\title{
REMARKS ON THE SIZE DISTRIBUTION OF COLLIDING AND FRAGMENTING PARTICLES
}

\author{
LOTHAR W. BANDERMANN \\ University of Hawaii
}

This paper is concerned with some aspects of determining the evolution of the size distribution of a finite number of mutually colliding and fragmenting particles such as the asteroids or interplanetary dust. If $n(m, t)$ is the number of particles per unit volume per mass interval at time $t$, then $\dot{n}=d n / d t$ is the rate at which that number changes with time. This rate can be calculated if the laws are known according to which the colliding bodies erode one another and fragment and if the influence of collisions on the motion of the particles is known. To reduce the complexity of the problem, one assumes that the speed of approach between the bodies is always the same $v_{\text {coll }}$ and that they, as well as the debris, occupy a fixed volume ("particles in a box"). Only collisions between two bodies are considered, and the way in which erosion and fragmentation occurs at a given value of $v_{\text {coll }}$ depends only on their masses. The particles are assumed to be spherical. One is particularly interested in stationary states (i.e., cases where $n$ can be factored into independent functions of $t$ and $m$ ):

$$
n(m, t)=T(t) \cdot \phi(m)
$$

and in steady states (i.e., where $d T / d t=0$ ). Steady states can of course be reached only over limited ranges of $m$ because no particles are supplied from outside to the system. Even for very simple assumed fragmentation laws, the equation for $\dot{n}$ is extremely complicated, being of a multiple integrodifferential type, and analytical solutions can be found (sometimes) for very restricted mass ranges and even then only by making some rather drastic approximations.

A simpler problem-namely, where the probability of destruction of a particle is independent of the total number and the mass distribution of the other particles in the system-was solved by Filippov (1961). Assuming that the probability is proportional to a power of the mass and that the size distribution of the fragments of a particle is given by a power law, he derived a formula by which the asymptotic $(t \rightarrow \infty)$ solution for $n$ can be calculated. In the collision problem, the probability of the collision depends on the relative 
numbers of other particles, and the size distribution of the fragments and erosion products depend on the masses of the colliding components. By neglecting the role of debris in further collisions, Piotrowski (1953) found a stationary solution with $\phi \propto m^{-5 / 3}$. Dohnanyi (1969), who did consider the role of debris in the evolution of the distribution, derived analytically a steady-state solution with $\phi \propto m^{-11 / 6}$, applicable to particles with intermediate sizes. Hellyer (1970) subsequently concluded that Piotrowski's law applies only to large masses when one considers the role of debris; i.e., to masses not covered by Dohnanyi's law. More recently, Dohnanyi (1970) has investigated the evolution of the large particles in a mass distribution, in particular, those particles that are not created by collisions of others; and he has concluded that their distribution function approaches asymptotically the $m^{-11 / 6}$ law. To appreciate the significance of these results, let us look at the equation for $n$ and at the fragmentation laws themselves.

There are two types of collisions. In erosive collisions the target mass $M$ is very much greater than the projectile mass $\mu$. (We assume that $M>\mu$.) As a result of the collision, a small amount of matter $M_{e}$ is eroded from the target mass $M$. For hypervelocity collisions that occur between asteroids as well as between interplanetary dust particles, $M_{e} \gg \mu$. Because $M_{e} \propto \mu$ (as experiments confirm (Gault, Shoemaker, and Moore, 1963)), for sufficiently large values of $\mu / M$, the mass $M$ will be disrupted. Those are explosive collisions.

The $m^{-11 / 6}$ law is determined almost exclusively by explosive collisions. The threshold projectile mass is equal to $M / \Gamma^{\prime}$, where the parameter $\Gamma^{\prime}$ depends on $v_{\text {coll }}$. A first approximation of the mass distribution of collisional debris derived from either type of collision is

$$
\frac{d N}{d m}=C m^{-\eta} d m
$$

where $\eta<2$. For erosive collisions, $\eta \cong 1.8, M_{e}=\Gamma \mu$, and the largest debris has a mass $M_{b}=\Lambda \mu ; \Gamma, \Gamma^{\prime}$, and $\Lambda$ are much greater than unity. These relations complete the erosive fragmentation law.

In the case of explosive collisions, the corresponding relations are much less well known, except $M_{e}=M+\mu$ in this case; $\eta$ is perhaps somewhat less than 1.8 (Gault, Shoemaker, and Moore, 1963; Gault and Wedekind, 1969). A relation for $M_{b}$ is unknown. Dohnanyi (1969) initially assumed $M_{b}$ to be proportional to $\mu$. This leads to absurd consequences, however; and he more recently suggested (Dohnanyi, 1970) that $M_{b}=\lambda M$, where $\lambda<1$.

It may be worthwhile at this time to consider qualitatively how the value of $M_{b}$ is affected by increasing or decreasing the masses $M$ and $\mu$. The kinetic energy in the center-of-mass rest frame is, per unit mass of the colliding particles,

$$
E \propto\left(\frac{M}{\mu}+2+\frac{\mu}{M}\right)^{-1}
$$


(See table I.) It appears that the entries in the first column of table I are inconsistent. Although the energy per unit mass in the center of mass increases sharply by increasing the projectile mass while keeping the target the same, the largest debris remains the same even though the decimation of the target must be increasingly severe. It is true, of course, that part of the energy is used for vaporization and for acceleration of fragments; glancing collisions may lead to a different $M_{b}$ than head-on collisions. But these effects are difficult to assess. It seems more reasonable to me to assume that $M_{b}$ decreases with increasing $\mu$, perhaps as follows:

$$
M_{b}=\lambda M\left(\frac{M}{\mu}\right)^{\delta}
$$

where $\lambda<1$ and, perhaps, $\delta \ll 1$. In such a case, we must require $\lambda\left(\Gamma^{\prime}\right)^{\delta} \leqslant 1$. The fragmentation laws for the two types of collisions are then given in table II.

Experiments indicate that $\Gamma, \Gamma^{\prime}$, and $\Lambda$ are proportional to $v_{\mathrm{coll}}{ }^{2}$. We expect $\lambda$ to decrease with increasing $v_{\text {coll }}$. If the smallest and largest masses in the system are $m_{<}$and $m_{>}$, respectively (particles with $m<m_{<}$in the case of the asteroid belt and interplanetary dust may be lost by the action of solar radiation pressure and radiation drag), the equation for $\dot{n}$ is

$$
\begin{aligned}
& \dot{n}(m, t)=-k n(m, t) \int_{\max \left(m_{<}, m / \Gamma^{\prime}\right)}^{m>}\left(m^{1 / 3}+\mu^{1 / 3}\right)^{2} n(\mu, t) d \mu \\
& -\kappa \frac{d}{d m}\left[n(m, t) \Gamma \int_{m_{<}}^{m / \Gamma^{\prime}} n(\mu, t) \mu\left(\mu^{1 / 3}+m^{1 / 3}\right)^{2} d \mu\right] \\
& +\kappa \int_{\max \left(m_{<} m / \Lambda\right)}^{m / \Gamma^{\prime}} n(\mu, t) d \mu \int_{\Gamma^{\prime} \mu}^{m_{>}}\left(\mu^{1 / 3}+M^{1 / 3}\right)^{2} \frac{d N}{d m} n(M, t) d M \\
& +\kappa \int_{\max \left[m_{<}, m / \lambda\left(\Gamma^{\prime}\right)^{\delta}\right]}^{m} n(\mu, t) d \mu \\
& \times \int_{\max \left[\mu,(m / \lambda)^{1 /(1+\delta)_{\mu} \delta /(1+\delta)}\right]}^{\min \left(m_{>}, \mu \Gamma^{\prime}\right)}\left(\mu^{1 / 3}+M^{1 / 3}\right)^{2} \frac{d N}{d m} n(M, t) d M
\end{aligned}
$$

The first and last terms are due to explosive collisions, the others to erosive collisions. The second term gives the change in $n$ caused by gradual erosion of large particles. (See Dohnanyi, 1969, for detailed discussions of collision equations.)

There are two ways to go about solving equation (3): substituting trial solutions of the stationary type, for instance, or starting with an initial 
TABLE I. - Effect of $\mathrm{M}$ and $\mu$ on Elements in a Collision System

\begin{tabular}{|c|c|c|c|c|}
\hline \multirow{2}{*}{ Element } & \multicolumn{3}{|c|}{$M>\mu$} & \multirow{2}{*}{$\frac{M=\mu}{\begin{array}{c}M \text { and } \mu \\
\text { increasing }\end{array}}$} \\
\hline & $\begin{array}{l}M \text { constant, } \\
\mu \text { increasing }\end{array}$ & $\begin{array}{c}\mu \text { constant, } \\
M \text { increasing }\end{array}$ & $\begin{array}{c}M / \mu \text { constant, } \\
M \text { and } \mu \text { increasing }\end{array}$ & \\
\hline $\begin{array}{l}M_{e} \\
E \\
M_{b} \propto M\end{array}$ & $\begin{array}{l}\text { Increases } \\
\text { Increases } \\
\text { Constant }\end{array}$ & $\begin{array}{l}\text { Increases } \\
\text { Decreases } \\
\text { Increases }\end{array}$ & $\begin{array}{l}\text { Increases } \\
\text { Constant } \\
\text { Increases }\end{array}$ & $\begin{array}{l}\text { Increases } \\
\text { Constant } \\
\text { Increases }\end{array}$ \\
\hline
\end{tabular}

TABLE II.--Fragmentation Laws

\begin{tabular}{c|c|c|c}
\hline Collisions & $M_{e}$ & \multicolumn{1}{|c|}{$M_{b}$} & \multicolumn{1}{c}{$C$} \\
\hline Erosive, $\mu<M / \Gamma^{\prime}$ & $\Gamma \mu$ & $\Lambda \mu$ & $(2-\eta) \Lambda^{\eta-2} \Gamma \mu^{\eta-1}$ \\
Explosive, $\mu>M / \Gamma^{\prime}$ & $M+\mu$ & $\lambda M(M / \mu)^{\delta}$ & $(2-\eta) \lambda^{\eta-2}(M+\mu) M^{(\eta-2)(1+\delta) \mu^{(2-\eta) \delta}}$ \\
\hline
\end{tabular}

distribution $n(m, 0)$ and recomputing again and again by determining $\Delta n$ for a given interval $\Delta t$. Because the right-hand side of equation (3) does not contain $t$ in explicit form, a stationary solution suggests itself naturally:

$$
\frac{d T / d t}{T^{2}}=-\frac{\kappa}{\phi}\left[\phi(m) \int_{\max \left(m_{<}, m / \Gamma^{\prime}\right)}^{m}\left(m^{1 / 3}+\mu^{1 / 3}\right)^{2} \phi(\mu) d \mu+\ldots\right]
$$

The power law $\phi \propto m^{-11 / 6}$ can be found by setting $\delta=0$, substituting a trial function $\phi \propto m^{-\alpha}$, setting the constant in equation (4) to zero (steady state), restricting $\alpha$ to a narrow range of possible values (in anticipation of the result), evaluating the integrals in equation (4), and discarding all but the two largest terms obtained for values of $m$ in the intermediate range. (See Dohnanyi, 1969.)

This procedure, however, raises questions concerning the uniqueness and stability of the solution and its relation to the general solution of equation (3); these questions I feel have not yet been answered satisfactorily. Quite strangely, the solution $\phi \propto \mathrm{m}^{-\alpha}$ does not seem to reflect any of the physics going into the problem (represented by the parameters $\Gamma$, etc.). Note also that the value of $\alpha$ is.very little different from the power index of the fragment size distribution $\eta$ (assumed to be equal to 1.8 for the above solution). This makes it impossible to decide, for instance, if the present asteroidal size distribution reflects the effects of the collisions having taken place over eons or the initial distribution formed quite recently in the fragmentation of a larger parent body. Another disadvantage is the lack of information about the actual 
evolution from a given initial distribution $n$. One would like to know, for instance, how long it takes to reach the steady state. There is no information about the large and the small particles. The solution for $T$ derived from equation (4) is of course just the asymptotic solution; therefore the initial development cannot be found by seeking a stationary solution for $n$. Finally, a collision equation (3) for particles with a narrow mass range (several orders of magnitude rather than several ten) does not lend itself to the above analysis. Instead, a numerical solution must be found by actually following the evolution of a distribution on a computer as it changes in the course of time, trying several sets of collision parameters $\Gamma$, etc.

A computational approach to solving the problem has the great advantage of allowing us to consider more accurate (and therefore often mathematically more complex) descriptions of the experimental data from which we build the fragmentation laws. ${ }^{1}$ The power-law substitution for $\phi(m)$ obviously is suggested by the fact that the collision probability of particles $M$ and $\mu$ is proportional to $\left(M^{1 / 3}+\mu^{1 / 3}\right)^{2}$ and also by the form of the suggested distribution of fragments. Other functions render an analytical approach all but hopeless. Just as analytical approaches are beset with special problems, so are computational ones. In the latter, $n$ becomes the number of particles with a fixed mass $m$ and is therefore an integer; however, when considering large ranges of mass, the number of the smallest particles is too large to be handled as an integer even on large computers such as the IBM 360, which the author used. Also, the time interval is discrete; and therefore one works with fractional events, noninteger numbers of particles, and, in general, nonconservation of total mass. If the chosen time interval is too large, then, in effect, the same particle is destroyed several times and debris is created from nonexistent particles. If it is too small, the evolution proceeds much too slowly (the computations become excessively expensive); if too large, then details in the evolution are lacking.

Some of the inaccuracy inherent in the numerical solution for $n(m, t)$ is balanced, however, by the great uncertainty in the fragmentation laws. The particles are not really spherical, of homogeneous composition, nor colliding head on at the same $v_{\text {coll }}$; and their occupied volume changes in the course of time for various reasons besides the effects of the collisions themselves. Mass is

${ }^{1}$ f find that the impact,experiment data of Gault and others (Gault, Shoemaker, and Moore, 1963; Gault and Wedekind, 1969) are better represented by

$$
\log \int^{M} m d N(m)=\text { const }+A \log m+B(\log m)^{2}
$$

The simple power law for $d N / d m$ is obtained by setting $B=0$. Otherwise one obtains a rather complicated form for $d N / d m$ :

$$
\frac{d N}{d m}=\text { const } m^{A-2}(A+2 B \log m) m^{B} \log m
$$


indeed actually lost from the system in the forms of both gas and very small particles, which are ejected from the system by radiation pressure or radiation drag. Finally, a collision does not necessarily lead only to erosion and fragmentation but could cause some aggregation of matter, though this is probably a minor effect.

I have recently begun numerical studies of the evolution of the particle size distribution under collisions, using an IBM 360 computer. Details of this program will be published elsewhere, and I would like to describe at this time some of the results. I first chose a narrow mass range (seven orders of magnitude) and assumed Dohnanyi's form of the fragmentation law $(\delta=0$; $\left.M_{b}=\lambda M\right)$ with $\Gamma^{\prime}=5000, \Gamma=100, \Lambda=10$, and $0.1<\lambda<1$. The ratio of masses $m_{i}$ and $m_{i+1}$ was set equal to $10^{0.1}$, and the number of the largest particles was 10. Various initial distributions $n$ were used. All were power laws suggested by the results of Dohnanyi and others. The time interval was adjusted during the program so that a "visible" change in $n$ could be detected. The number of collisions between two given species of particles as well as the number of particles created by collisions were rounded to integers using a random number generator and a uniform probability distribution on $(0,1)$. This rounding insured that no "ghost" particles and/or fragments appeared in the problem, and it was, of course, necessary because of the discrete masses $m_{i}$ of the program. The total mass decreased with time because debris with a mass less than a certain mass $m_{1}$ was presumed to be lost from the system. Under these conditions, I found that, regardless of the value of $\lambda$ or of the initial distribution, particles with intermediate sizes were immediately and, eventually, greatly diminished in number as compared with very small or very large particles. A stationary state was reached eventually in this size range that was fairly well represented by a power law with index $\alpha \cong 3$. Because the number of particles of all sizes eventually decreased with time, the particles with intermediate size eventually disappeared altogether leaving a bimodal size distribution. For $\delta=1$, the loss of particles with intermediate masses occurred without first reaching a stationary state described above. The two modes of the final distribution were not at any time given by power laws. The preliminary result of studies of the collisions between masses ranging over 30 orders of magnitude (corresponding to asteroidal radii from $10 \mu \mathrm{m}$ to $100 \mathrm{~km}$ ) gave a quite different result. I found that the number of very large particles is quickly diminished in relation to intermediate size or small particles.

These results must be tested by further calculations using many sets of parameters and initial distributions before it becomes worthwhile to formulate them in a more quantitative manner. I should like to mention, nevertheless, that the results suggest the existence of a bimodal size distribution for interplanetary dust: some small particles, some large particles, but nothing in between, to put it in simple words. This may explain some of the disagreement concerning the dust size distribution as determined by different experiments (e.g., polarimetry versus particle-impact counting). 


\section{ACKNOWLEDGMENT}

This research was supported by NSF Grant GA-10 883.

\section{REFERENCES}

Dohnanyi, J. S. 1969, Collisional Model of Asteroids and Their Debris. J. Geophys. Res. 74, 2531.

Dohnanyi, J. S. 1970, Mass Distribution of Asteroids. Bellcomm TM-70-2015-6.

Filippov, A. F. 1961, Theory of Probability, vol. 6, p. 275.

Gault, D. E., Shoemaker, E. M., and Moore, H. J. 1963, Fragments Ejected From Lunar Surface by Meteoroid Impact Analyzed on Basis of Studies of Hypervelocity Impact in Rock and Sand. NASA TN-D 1767.

Gault, D. E., and Wedekind, J. A. 1969, The Destruction of Tektites by Micrometeoroid Impact. J. Geophys. Res. 74, 6780.

Hellyer, B. 1970, The Fragmentation of the Asteroids. Mon. Notic. Roy. Astron. Soc. 148, 383.

Piotrowski, S. 1953, The Collisions of Asteroids. Acta Astron. Ser. A 5, 115-138.

\section{DISCUSSION}

DOHNANYI: It is my experience that attempts to solve the collision equation of my model by successive approximations may involve convergence difficulties. I wonder if this is responsible for the differences in our results?

BANDERMANN: If the time interval $\Delta t$ for the successive steps is chosen reasonably small, then I have found little or no dependence of the eventual evolution of the distribution on $\Delta t$, although the first few steps may show a strange behavior of the distribution. 